\title{
Peptide derivatives of retinylamine prevent retinal degeneration with minimal side effects on vision in mice
}

Guanping Yu ${ }^{1}$, Song-Qi Gao ${ }^{1}$, Zhiqian Dong ${ }^{2}$, Li Sheng ${ }^{1}$, Da Sun ${ }^{1}$, Ning Zhang ${ }^{3}$, Jianye Zhang ${ }^{2}$, Seunghee Margeivicus $^{4}$, Pingfu Fu ${ }^{4}$, Marcin Golczak ${ }^{3}$, Akiko Maeda ${ }^{2,5}$, Krzysztof Palczewski ${ }^{2 *}$, Zheng-Rong Lu ${ }^{{ }^{*}}$

${ }^{1}$ Department of Biomedical Engineering, School of Engineering, Case Western Reserve University, Cleveland, Ohio 44106

${ }^{2}$ Gavin Herbert Eye Institute, Departments of Ophthalmology, Physiology \& Biophysics, and Chemistry, University of California, Irvine, CA 92697

${ }^{3}$ Department of Pharmacology, School of Medicine, Case Western Reserve University, Cleveland, Ohio 44106

${ }^{4}$ Department of Epidemiology and Biostatistics, School of Medicine, Case Western Reserve University, Cleveland, Ohio 44106, USA

${ }^{5}$ Department of Ophthalmology, School of Medicine, Case Western Reserve University, Cleveland, Ohio 44106, USA

- Synthesis of Ret-NH $\mathrm{NH}_{2}$ derivatives and emixustat-VG

- References

- Table of Ret- $\mathrm{NH}_{2}$ derivatives

- $\mathrm{RVG}$ and Ret-NH $\mathrm{NH}_{2}$ Stability for storage

- LC-MS analysis of the Ret- $\mathrm{NH}_{2}$ derivatives from the eye extraction

- HPLC analysis of Ret-NH$H_{2}$ derivatives

- MALDI-TOF MS

- ${ }^{1} \mathrm{H}$ NMR and ${ }^{13} \mathrm{C}$ NMR spectra 


\section{Synthesis of Ret-NH2 derivatives and emixustat-VG}

All commercially available reagents and solvent were analytically pure substances and used as received. Reactions were monitored by thin-layer chromatography (TLC) on silica gel plates (60 $\left.F_{254}\right)$ with a fluorescent indicator, and independently visualized with UV light. Intermediates and target Ret- $\mathrm{NH}_{2}$ derivatives were purified by column chromatography on silica (Silica gel grade: 200-400 mesh, 40-63 $\mu \mathrm{m})$. All new compounds gave spectroscopic analyses ( $\left.{ }^{1} \mathrm{H} \mathrm{NMR},{ }^{13} \mathrm{C} \mathrm{NMR}\right)$ consistent with their expected structures. NMR spectra were recorded at $400 \mathrm{MHz}$ in $\mathrm{CDCl}_{3}$ or Acetone- $d_{6}$ and chemical shift values $(\delta)$ are given in ppm. ${ }^{1} \mathrm{H}$ NMR spectra are reported in parts per million $(\delta)$ relative to the residual (indicated) solvent peak. Data for ${ }^{1} \mathrm{H}$ NMR are reported as follows: chemical shift $(\delta \mathrm{ppm})$, multiplicity $(\mathrm{s}=$ singlet, bs = broad singlet, $\mathrm{d}=$ doublet, $\mathrm{t}=$ triplet, $\mathrm{q}=$ quartet, $\mathrm{dd}=$ double doublet, $\mathrm{ddd}=$ double double doublet, $\mathrm{m}=$ multiplet, $\mathrm{cm}=$ complex multiplet); integration, and coupling constants in $\mathrm{Hz} .{ }^{13} \mathrm{C}$ NMR spectra were obtained on $400 \mathrm{MHz}$ spectrometers $(100 \mathrm{MHz}$ actual frequency) and are reported in parts per million $(\delta)$ relative to the residual (indicated) solvent peak. Matrix-assisted laser desorption/ionization time-of-flight (MALDI-TOF) mass spectra were acquired on a Bruker Autoflex III MALDI-TOF MS in a linear mode with 2,5-dihydroxybenzoic acid (2,5-DHB) as the matrix. Ion-pairing reverse-phase HPLC (RP-HPLC) was performed on an Agilent 1100 series HPLC system fitted with a Beckman Coulter Ultrasphere ODS $4.6 \mathrm{~mm} \times 25 \mathrm{~cm}, 5 \mu \mathrm{m}$ pore size column. HPLC conditions: mobile phase of $\mathrm{H}_{2} \mathrm{O}$ : acetonitrile $(40: 60, v: v)$ with $0.05 \%$ trifluoroacetic acid; flow rate of $1.0 \mathrm{~mL} / \mathrm{min}$ and $\mathrm{UV}$ detector set at $325 \mathrm{~nm}$.

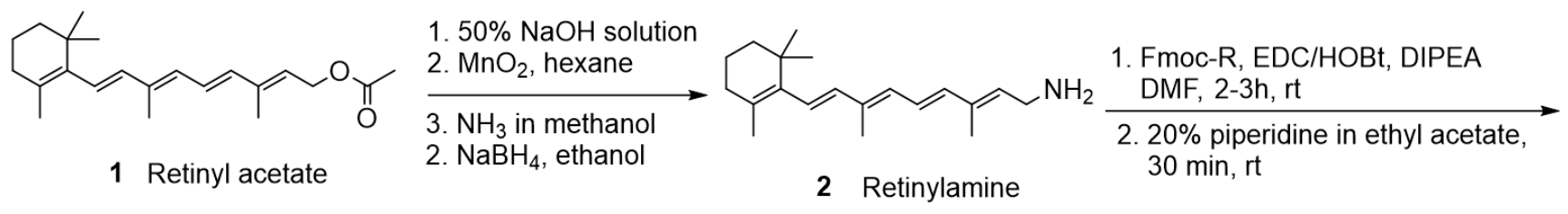

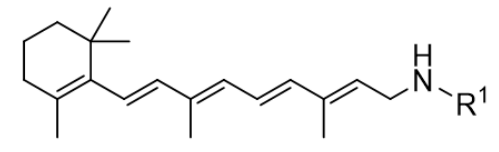

3 Retinylamine derivertives
$\mathrm{R}^{1}$ = L-Alanine; L-Phenylalanine; Glycine; L-Isoleucine; L-Leucine; L-Proline; L-Valine; D-Alaline; D-Valine; $\beta$-Alanine; Pregabalin; L-Valine-Glycine; Glycine-L-Valine; Glycine-Glycine;

Fig. S1. Synthetic scheme for the Ret- $\mathrm{NH}_{2}$ derivatives. 


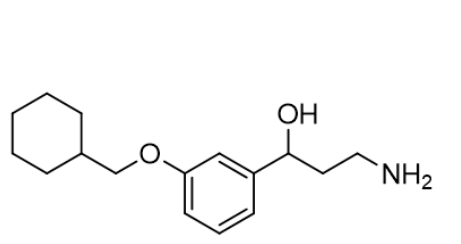

racemic emixustat
1. Fmoc-Gly-Val, EDC/HOBt, DIPEA

$\mathrm{DMF}, 2 \mathrm{~h}, \mathrm{rt}$

2. $10 \% \mathrm{~K}_{2} \mathrm{CO}_{3}$ aqueous solution, $1 \mathrm{~h}$, rt

3. $20 \%$ piperidine in ethyl acetate,

$30 \mathrm{~min}, \mathrm{rt}$

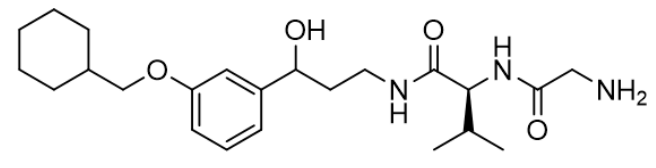

emixustat-L-Val-Gly (EVG)

Fig. S2. Synthetic scheme for emixustat-L-Val-Gly (EVG).

\section{Synthesis of Ret-NH 2 Derivatives and emixustat-L-Val-Gly (EVG)}

Ret- $\mathrm{NH}_{2}$ was synthesized and purified according to the procedure published by Golczak et al ${ }^{l}$. (2E,4E,6E,8E)-3,7-dimethyl-9-(2,6,6-trimethylcyclohex-1-en-1-yl)nona-2,4,6,8-tetraen-1-amine (2, Ret$\mathrm{NH}_{2}$ ): yield 23.5\%; ${ }^{1} \mathrm{H}$ NMR (400 MHz, $\left.\mathrm{CDCl}_{3}\right) \delta 6.56(\mathrm{dd}, J=15.2,11.3 \mathrm{~Hz}, 1 \mathrm{H}), 6.27(\mathrm{~d}, J=15.1 \mathrm{~Hz}$, $1 \mathrm{H}), 6.21-6.04(\mathrm{~m}, 3 \mathrm{H}), 5.58(\mathrm{t}, J=6.9 \mathrm{~Hz}, 1 \mathrm{H}), 3.47(\mathrm{t}, J=2.8 \mathrm{~Hz}, 2 \mathrm{H}), 2.92(\mathrm{~s}, 2 \mathrm{H}), 2.01(\mathrm{t}, J=6.3$ $\mathrm{Hz}, 2 \mathrm{H}), 1.94$ (d, $J=4.5 \mathrm{~Hz}, 3 \mathrm{H}), 1.84(\mathrm{~d}, J=7.1 \mathrm{~Hz}, 3 \mathrm{H}), 1.70$ (t, $J=3.4 \mathrm{~Hz}, 3 \mathrm{H}), 1.65-1.56(\mathrm{~m}, 2 \mathrm{H})$, $1.49-1.41(\mathrm{~m}, 2 \mathrm{H}), 1.02(\mathrm{~s}, 6 \mathrm{H})$. MALDI-TOF $m / z$ [M-NH$]^{+}$calcd. for $\mathrm{C}_{20} \mathrm{H}_{18}: 268.219$, found: 268.857.

The synthetic scheme for Ret- $\mathrm{NH}_{2}$ derivatives is shown in Fig.1 (above). A $100 \mathrm{~mL}$ flask was charged under an atmosphere of nitrogen with Fmoc- $\mathrm{R}^{1}$ (2.0 mmol, 1.0 equiv.), 1-Ethyl-3-(3dimethylaminopropyl)carbodiimide (EDC) (383 mg, $2.0 \mathrm{mmol}, 1.0$ equiv.), hydroxybenzotriazole (HOBt) (270 mg, $2.0 \mathrm{mmol}, 1.0$ equiv.), N, N- diisopropylethylamine (517 mg, $4.0 \mathrm{mmol}, 2.0$ equiv.), and Ret$\mathrm{NH}_{2}$ (571 mg, $2.0 \mathrm{mmol}, 1.0$ equiv.) dissolved in dry DMF (20 mL) at room temperature. Stirring was continued at room temperature for 2-3 $\mathrm{h}$; then the reaction was quenched with water. The intermediate Fmoc-protected Ret- $\mathrm{NH}_{2}$ derivative (Ret- ${ }^{1}$-Fmoc) was extracted by ethyl acetate and washed with brine and dried over $\mathrm{Na}_{2} \mathrm{SO}_{4}$. The crude product was purified by silica gel column chromatography, with hexane and ethyl acetate as the eluant. $10 \mathrm{~mL} 20 \%$ piperidine in ethyl acetate was added to Ret- $\mathrm{R}^{1}$-Fomc to remove Fmoc. The crude product was purified by silica gel column chromatography, with ethyl acetate and methanol plus $5 \% \mathrm{NH}_{3}$ as the eluant.

(S)-2-amino- $N$-((2E,4E,6E,8E)-3,7-dimethyl-9-(2,6,6-trimethylcyclohex-1-en-1-yl)nona-2,4,6,8tetraen-1-yl)propanamide (3a, Ret-L-Ala): yield 17.5\% (125 mg); ${ }^{1} \mathrm{H}$ NMR (400 MHz, Acetone- $\left.d_{6}\right) \delta 6.63$ (dd, $J=15.1,11.3 \mathrm{~Hz}, 1 \mathrm{H}), 6.29$ (d, $J=15.2 \mathrm{~Hz}, 1 \mathrm{H}), 6.20-6.05(\mathrm{~m}, 3 \mathrm{H}), 5.51(\mathrm{t}, J=6.8 \mathrm{~Hz}, 1 \mathrm{H}), 3.97$ (d, $J=7.0 \mathrm{~Hz}, 2 \mathrm{H}), 3.88$ (q, $J=7.0 \mathrm{~Hz}, 1 \mathrm{H}), 2.00$ (t, $J=6.2 \mathrm{~Hz}, 2 \mathrm{H}), 1.94$ (s, 3H), 1.85 (s, 3H), 1.69 (s, $3 \mathrm{H}), 1.64-1.56(\mathrm{~m}, 2 \mathrm{H}), 1.49-1.41(\mathrm{~m}, 2 \mathrm{H}), 1.17$ (d, J=7.0 Hz, 3H), $1.00(\mathrm{~s}, 6 \mathrm{H}) .{ }^{13} \mathrm{C} \mathrm{NMR}(100 \mathrm{MHz}$, 
Acetone- $\left.d_{6}\right) \delta 173.4,138.2,138.0,137.1,136.3,135.5,130.9,129.4,128.9,126.3,124.6,59.2,39.7,36.8$, 34.2, 33.0, 28.7, 21.4, 19.3, 19.0, 12.2, 12.1. MALDI-TOF $m / z, \mathrm{M}^{+}$calcd. for $\mathrm{C}_{23} \mathrm{H}_{36} \mathrm{~N}_{2} \mathrm{O}$ : 356.283; found: 355.788 .

(S)-2-amino- $N$-((2E,4E,6E,8E)-3,7-dimethyl-9-(2,6,6-trimethylcyclohex-1-en-1-yl)nona-2,4,6,8tetraen-1-yl)-3-phenylpropanamide (3b, Ret-L-Phe): yield 38.5\% (333 mg); ${ }^{1} \mathrm{H}$ NMR (400 MHz, Acetone$\left.d_{6}\right) \delta 7.37-7.00(\mathrm{~m}, 6 \mathrm{H}), 6.64(\mathrm{dd}, J=15.1,11.3 \mathrm{~Hz}, 1 \mathrm{H}), 6.29(\mathrm{~d}, J=15.1 \mathrm{~Hz}, 1 \mathrm{H}), 6.23-6.02(\mathrm{~m}, 3 \mathrm{H})$, $5.47(\mathrm{t}, J=6.8 \mathrm{~Hz}, 1 \mathrm{H}), 4.14-3.85(\mathrm{~m}, 2 \mathrm{H}), 3.20(\mathrm{dd}, J=12.9,3.4 \mathrm{~Hz}, 1 \mathrm{H}), 2.85-2.66(\mathrm{~m}, 1 \mathrm{H}), 2.01$ $(\mathrm{dd}, J=10.3,4.0 \mathrm{~Hz}, 2 \mathrm{H}), 1.95(\mathrm{~s}, 3 \mathrm{H}), 1.86(\mathrm{~s}, 3 \mathrm{H}), 1.69(\mathrm{~s}, 3 \mathrm{H}), 1.60(\mathrm{ddd}, J=8.8,8.0,4.5 \mathrm{~Hz}, 2 \mathrm{H})$, $1.50-1.41(\mathrm{~m}, 2 \mathrm{H}), 1.02(\mathrm{~s}, 6 \mathrm{H}) .{ }^{13} \mathrm{C} \mathrm{NMR}\left(100 \mathrm{MHz}\right.$, Acetone- $\left.d_{6}\right) \delta 172.1,138.8,138.19,138.0,137.1$, 136.3, 135.5, 131.0, 130.1, 129.3, 128.9, 128.2, 126.4, 126.3, 124.6, 65.9, 40.7, 39.7, 36.9, 34.7, 33.0, 28.8, 21.5, 19.4, 12.3, 12.2. MALDI-TOF $m / z$ [M+Na $]^{+}$calcd. for $\mathrm{C}_{29} \mathrm{H}_{40} \mathrm{~N}_{2} \mathrm{ONa}$ : 455.304; found: 454.833 . 2-amino- $N-((2 E, 4 E, 6 E, 8 E)-3,7-d i m e t h y 1-9-(2,6,6-t r i m e t h y l c y c l o h e x-1-e n-1-y 1) n o n a-2,4,6,8-t e t r a e n-1-$ yl)acetamide (3c, Ret-Gly): yield 23.7\% (162 mg); ${ }^{1} \mathrm{H}$ NMR (400 MHz, Acetone- $\left.d_{6}\right) \delta 7.81(\mathrm{~s}, 1 \mathrm{H}), 6.64$ $(\mathrm{dd}, J=15.1,11.2 \mathrm{~Hz}, 1 \mathrm{H}), 6.30(\mathrm{~d}, J=15.1 \mathrm{~Hz}, 1 \mathrm{H}), 6.24-6.05(\mathrm{~m}, 3 \mathrm{H}), 5.53(\mathrm{t}, J=6.9 \mathrm{~Hz}, 1 \mathrm{H}), 4.03$ $(\mathrm{dd}, J=6.4,5.3 \mathrm{~Hz}, 2 \mathrm{H}), 3.71(\mathrm{~s}, 2 \mathrm{H}), 3.22(\mathrm{~s}, 2 \mathrm{H}), 2.00(\mathrm{t}, J=6.2 \mathrm{~Hz}, 2 \mathrm{H}), 1.95$ (s, 3H), 1.87 (s, 3H), $1.69(\mathrm{~s}, 3 \mathrm{H}), 1.64-1.55(\mathrm{~m}, 2 \mathrm{H}), 1.49-1.40(\mathrm{~m}, 2 \mathrm{H}), 1.01(\mathrm{~s}, 6 \mathrm{H}) .{ }^{13} \mathrm{C}$ NMR $\left(100 \mathrm{MHz}\right.$, Acetone- $\left.d_{6}\right) \delta$ 170.2 , 138.2, 138.0, 137.1, 136.3, 135.5, 130.9, 129.3, 128.9, 126.3, 124.6, 54.3, 39.7, 36.7, 34.2, 33.0, 28.7, 21.4, 19.3, 12.2, 12.1. MALDI-TOF $m / z \mathrm{M}^{+}$calcd. for $\mathrm{C}_{22} \mathrm{H}_{34} \mathrm{~N}_{2} \mathrm{O}$ : 342.267; found: 342.792 .

(2S,3S)-2-amino- $N$-((2E,4E,6E,8E)-3,7-dimethyl-9-(2,6,6-trimethylcyclohex-1-en-1-yl)nona-2,4,6,8tetraen-1-yl)-3-methylpentanamide (3d, Ret-L-Ile): yield 31.2\% (249 mg); ${ }^{1} \mathrm{H}$ NMR (400 MHz, Acetoned6) $\delta 6.63(\mathrm{dd}, J=14.9,11.3 \mathrm{~Hz}, 1 \mathrm{H}), 6.29(\mathrm{~d}, J=15.1 \mathrm{~Hz}, 1 \mathrm{H}), 6.12(\mathrm{dd}, J=16.9,10.0 \mathrm{~Hz}, 3 \mathrm{H}), 5.51(\mathrm{t}$, $J=6.6 \mathrm{~Hz}, 1 \mathrm{H}), 4.11-3.76(\mathrm{~m}, 3 \mathrm{H}), 2.09-2.01(\mathrm{~m}, 2 \mathrm{H}), 1.95(\mathrm{~s}, 3 \mathrm{H}), 1.86(\mathrm{~s}, 3 \mathrm{H}), 1.69(\mathrm{~s}, 3 \mathrm{H}), 1.63-$ $1.56(\mathrm{~m}, 2 \mathrm{H}), 1.49-1.40(\mathrm{~m}, 2 \mathrm{H}), 1.22-1.14(\mathrm{~m}, 2 \mathrm{H}), 0.99$ (d, $J=13.4 \mathrm{~Hz}, 6 \mathrm{H}), 0.85(\mathrm{dd}, J=11.9,7.1$ $\mathrm{Hz}, 6 \mathrm{H}) .{ }^{13} \mathrm{C}$ NMR $\left(100 \mathrm{MHz}\right.$, Acetone- $\left.d_{6}\right) \delta 171.7,138.2,138.0,137.1,136.2,135.4,130.9,129.4,128.9$, $126.3,124.5,68.7,40.3,39.7,36.7,34.2,32.9,28.7,25.3,21.4,19.3,15.4,12.2,12.1,11.7$. MALDI-TOF $m / z \mathrm{M}^{+}$calcd. for $\mathrm{C}_{26} \mathrm{H}_{42} \mathrm{~N}_{2} \mathrm{O}: 398.330$; found: 398.838 .

(S)-2-amino- $N$-((2E,4E,6E,8E)-3,7-dimethyl-9-(2,6,6-trimethylcyclohex-1-en-1-yl)nona-2,4,6,8tetraen-1-yl)-4-methylpentanamide (3e, Ret-L-Leu): yield 26.8\% (214 mg); ${ }^{1} \mathrm{H}$ NMR (400 MHz, Acetone$\left.d_{6}\right) \delta 6.63(\mathrm{dd}, J=15.1,11.3 \mathrm{~Hz}, 1 \mathrm{H}), 6.29(\mathrm{~d}, J=15.2 \mathrm{~Hz}, 1 \mathrm{H}), 6.24-6.02(\mathrm{~m}, 3 \mathrm{H}), 5.50(\mathrm{t}, J=6.9 \mathrm{~Hz}$, 
1H), $4.12-3.88(\mathrm{~m}, 3 \mathrm{H}), 2.00(\mathrm{~m}, 2 \mathrm{H}), 1.94(\mathrm{~s}, 3 \mathrm{H}), 1.85(\mathrm{~s}, 3 \mathrm{H}), 1.69(\mathrm{~s}, 3 \mathrm{H}), 1.63-1.55(\mathrm{~m}, 4 \mathrm{H}), 1.50$ $-1.40(\mathrm{~m}, 3 \mathrm{H}), 1.01(\mathrm{~s}, 6 \mathrm{H}), 0.87(\mathrm{dd}, J=13.8,6.3 \mathrm{~Hz}, 6 \mathrm{H}) .{ }^{13} \mathrm{C}$ NMR $\left(100 \mathrm{MHz}\right.$, Acetone- $\left.d_{6}\right) \delta 172.8$, 138.2, 138.0, 137.1, 136.2, 135.4, 130.9, 129.4, 128.9, 126.3, 124.5, 63.0, 44.1, 39.7, 36.8, 34.2, 32.9, 28.7, 24.6, 22.9, 22.3, 21.4, 19.3, 12.2, 12.0. MALDI-TOF $m / z, \mathrm{M}^{+}$, calcd. for $\mathrm{C}_{26} \mathrm{H}_{42} \mathrm{~N}_{2} \mathrm{O}$ : 398.330; found: 398.809 .

(S)-N-((2E,4E,6E,8E)-3,7-dimethyl-9-(2,6,6-trimethylcyclohex-1-en-1-yl)nona-2,4,6,8-tetraen-1yl)pyrrolidine-2-carboxamide (3f, Ret-L-Pro): yield 20.6\% (158 mg); ${ }^{1} \mathrm{H}$ NMR (400 MHz, Acetone- $\left.d_{6}\right) \delta$ $6.63(\mathrm{dd}, J=15.1,11.3 \mathrm{~Hz}, 1 \mathrm{H}), 6.29(\mathrm{~d}, J=15.1 \mathrm{~Hz}, 1 \mathrm{H}), 6.20-6.04(\mathrm{~m}, 3 \mathrm{H}), 5.51(\mathrm{t}, J=6.9 \mathrm{~Hz}, 1 \mathrm{H})$, $3.93(\mathrm{~d}, J=7.0 \mathrm{~Hz}, 2 \mathrm{H}), 3.65(\mathrm{dd}, J=8.8,5.5 \mathrm{~Hz}, 1 \mathrm{H}), 2.88(\mathrm{ddt}, J=22.8,10.3,6.5 \mathrm{~Hz}, 2 \mathrm{H}), 2.00(\mathrm{~d}, J$ $=5.2 \mathrm{~Hz}, 2 \mathrm{H}), 1.94(\mathrm{~s}, 3 \mathrm{H}), 1.86(\mathrm{~s}, 3 \mathrm{H}), 1.69(\mathrm{~s}, 3 \mathrm{H}), 1.66-1.57(\mathrm{~m}, 6 \mathrm{H}), 1.48-1.43(\mathrm{~m}, 2 \mathrm{H}), 1.01(\mathrm{~s}$, $6 \mathrm{H}) .{ }^{13} \mathrm{C}$ NMR (100 MHz, Acetone- $\left.d_{6}\right) \delta$ 174.8, 138.2, 138.0, 137.1, 136.3, 135.4, 130.9, 129.3, 128.9, $126.3,124.6,60.7,47.1,39.7,36.9,34.3,33.0,30.8,28.8,26.2,21.5,19.4,12.3,12.2$. MALDI-TOF $m / z$ $\mathrm{M}^{+}$calcd. for $\mathrm{C}_{25} \mathrm{H}_{38} \mathrm{~N}_{2} \mathrm{O}: 382.298$; found: 382.711 .

(S)-2-amino- $N$-((2E,4E,6E,8E)-3,7-dimethyl-9-(2,6,6-trimethylcyclohex-1-en-1-yl)nona-2,4,6,8tetraen-1-yl)-3-methylbutanamide (3g, Ret-L-Val): yield 22.4\% (172 mg); $\left.{ }^{1} \mathrm{H} \mathrm{NMR} \mathrm{(400} \mathrm{MHz,} \mathrm{CDCl}_{3}\right) \delta$ $7.32(\mathrm{t}, J=5.2 \mathrm{~Hz}, 1 \mathrm{H}), 6.50(\mathrm{dd}, J=15.1,11.3 \mathrm{~Hz}, 1 \mathrm{H}), 6.18(\mathrm{~d}, J=15.1 \mathrm{~Hz}, 1 \mathrm{H}), 6.08-5.91(\mathrm{~m}, 3 \mathrm{H})$, $5.41(\mathrm{t}, J=7.0 \mathrm{~Hz}, 1 \mathrm{H}), 3.94(\mathrm{t}, J=6.3 \mathrm{~Hz}, 2 \mathrm{H}), 3.16(\mathrm{~d}, J=3.9 \mathrm{~Hz}, 1 \mathrm{H}), 2.19(\mathrm{~m}, 2 \mathrm{H}), 1.94(\mathrm{~s}, 3 \mathrm{H}), 1.87$ $(\mathrm{s}, 3 \mathrm{H}), 1.79(\mathrm{~s}, 3 \mathrm{H}), 1.63(\mathrm{~s}, 3 \mathrm{H}), 1.53(\mathrm{~m}, 2 \mathrm{H}), 1.43-1.32(\mathrm{~m}, 2 \mathrm{H}), 0.94(\mathrm{~s}, 6 \mathrm{H}), 0.91(\mathrm{~d}, J=7.0 \mathrm{~Hz}$ $3 \mathrm{H}), 0.75(\mathrm{~d}, J=6.8 \mathrm{~Hz}, 3 \mathrm{H}) .{ }^{13} \mathrm{C} \mathrm{NMR}\left(100 \mathrm{MHz}, \mathrm{CDCl}_{3}\right) \delta 174.3,138.0,137.8,137.2,136.4,136.2$, 130.3, 129.4, 127.5, 126.8, 124.9, 60.3, 39.8, 37.4, 34.4, 33.2, 31.1, 29.1, 21.9, 19.9, 19.4, 16.4, 12.9, 12.8. MALDI-TOF $m / z \mathrm{M}^{+}$calcd. for $\mathrm{C}_{25} \mathrm{H}_{40} \mathrm{~N}_{2} \mathrm{O}$ : 384.314 ; found: 384.750 .

(R)-2-amino- $N$-((2E,4E,6E,8E)-3,7-dimethyl-9-(2,6,6-trimethylcyclohex-1-en-1-yl)nona-2,4,6,8tetraen-1-yl)propanamide (3h, Ret-D-Ala): yield 7.9\% (56 mg); ${ }^{1} \mathrm{H}$ NMR (400 MHz, Acetone- $\left.d_{6}\right) \delta 6.62$ $(\mathrm{dd}, J=15.0,11.2 \mathrm{~Hz}, 1 \mathrm{H}), 6.28(\mathrm{~d}, J=15.2 \mathrm{~Hz}, 1 \mathrm{H}), 6.19-6.00(\mathrm{~m}, 3 \mathrm{H}), 5.49$ (t, $J=6.9 \mathrm{~Hz}, 1 \mathrm{H}), 3.96$ $(\mathrm{t}, J=5.6 \mathrm{~Hz}, 2 \mathrm{H}), 3.85(\mathrm{q}, J=6.9 \mathrm{~Hz}, 1 \mathrm{H}), 1.98(\mathrm{t}, J=6.3 \mathrm{~Hz}, 2 \mathrm{H}), 1.93(\mathrm{~d}, J=1.0 \mathrm{~Hz}, 3 \mathrm{H}), 1.84(\mathrm{~d}, J$ $=1.0 \mathrm{~Hz}, 3 \mathrm{H}), 1.67(\mathrm{~d}, J=0.8 \mathrm{~Hz}, 3 \mathrm{H}), 1.62-1.54(\mathrm{~m}, 2 \mathrm{H}), 1.48-1.40(\mathrm{~m}, 2 \mathrm{H}), 1.14(\mathrm{~d}, J=7.0 \mathrm{~Hz}, 3 \mathrm{H})$, $0.99(\mathrm{~s}, 6 \mathrm{H}) .{ }^{13} \mathrm{C}$ NMR $\left(100 \mathrm{MHz}\right.$, Acetone- $\left.d_{6}\right) \delta 173.3,138.2,138.0,137.1,136.2,135.4,130.9,129.4$, $128.9,126.3,124.6,59.2,39.7,36.7,34.2,32.9,28.7,21.3,19.3,18.9,12.1,12.0$. MALDI-TOF $m / z \mathrm{M}^{+}$ calcd. for $\mathrm{C}_{23} \mathrm{H}_{36} \mathrm{~N}_{2} \mathrm{O}: 356.283$; found: 356.694 . 
(R)-2-amino- $N$-((2E,4E,6E,8E)-3,7-dimethyl-9-(2,6,6-trimethylcyclohex-1-en-1-yl)nona-2,4,6,8tetraen-1-yl)-3-methylbutanamide (3i, Ret-D-Val): yield 17.3\% (133 mg); ${ }^{1} \mathrm{H}$ NMR (400 MHz, Acetone$\left.d_{6}\right) \delta 6.61(\mathrm{dd}, J=15.1,11.3 \mathrm{~Hz}, 1 \mathrm{H}), 6.27(\mathrm{~d}, J=15.2 \mathrm{~Hz}, 1 \mathrm{H}), 6.17-6.02(\mathrm{~m}, 3 \mathrm{H}), 5.49$ (t, $J=6.9 \mathrm{~Hz}$, 1H), $4.07-3.84(\mathrm{~m}, 2 \mathrm{H}), 3.72$ (d, $J=4.6 \mathrm{~Hz}, 1 \mathrm{H}), 3.45-3.08$ (m, 2H), 1.98 (d, $J=6.0 \mathrm{~Hz}, 2 \mathrm{H}), 1.92$ (d, $J=0.8 \mathrm{~Hz}, 3 \mathrm{H}), 1.84$ (d, $J=0.9 \mathrm{~Hz}, 3 \mathrm{H}), 1.66$ (t, $J=1.6 \mathrm{~Hz}, 3 \mathrm{H}), 1.61-1.55$ (m, 2H), $1.48-1.40$ (m, 2H), 0.99 (s, 6H), 0.83 (dd, $J=14.7,6.9 \mathrm{~Hz}, 6 \mathrm{H}) .{ }^{13} \mathrm{C}$ NMR (100 MHz, Acetone- $\left.d_{6}\right) \delta 171.81,138.17$, $137.98,137.13,136.11,135.40,130.90,129.47,128.84,126.26,124.51,69.48,39.66,36.68,34.21,33.22$, $32.85,28.68,21.35,19.29,18.98,17.79,12.16,12.04$. MALDI-TOF $m / z[\mathrm{M}+\mathrm{Na}]^{+}$calcd. for $\mathrm{C}_{25} \mathrm{H}_{40} \mathrm{~N}_{2} \mathrm{ONa}$ : 407.304; found: 406.704 .

3-amino- $N$-((2E,4E,6E,8E)-3,7-dimethyl-9-(2,6,6-trimethylcyclohex-1-en-1-yl)nona-2,4,6,8-tetraen-1yl)propanamide (3j, Ret- $\beta$-Ala): yield 14.0\% (100 mg); ${ }^{1} \mathrm{H}$ NMR (400 MHz, Acetone- $\left.d_{6}\right) \delta 6.62$ (dd, $J=$ $15.1,11.3 \mathrm{~Hz}, 1 \mathrm{H}), 6.28(\mathrm{dd}, J=15.2,8.4 \mathrm{~Hz}, 1 \mathrm{H}), 6.21-6.01(\mathrm{~m}, 3 \mathrm{H}), 5.62-5.43(\mathrm{~m}, 1 \mathrm{H}), 3.90(\mathrm{~d}, J=$ $6.9 \mathrm{~Hz}, 2 \mathrm{H}), 3.39$ (t, $J=6.8 \mathrm{~Hz}, 2 \mathrm{H}), 2.38(\mathrm{t}, J=6.8 \mathrm{~Hz}, 2 \mathrm{H}), 1.98$ (d, $J=6.2 \mathrm{~Hz}, 2 \mathrm{H}), 1.92(\mathrm{~s}, 3 \mathrm{H}), 1.83$ (d, $J=0.9 \mathrm{~Hz}, 3 \mathrm{H}), 1.67$ (d, $J=0.8 \mathrm{~Hz}, 3 \mathrm{H}), 1.57$ (td, $J=6.3,2.6 \mathrm{~Hz}, 2 \mathrm{H}), 1.47-1.40$ (m, 2H), 0.99 (s, 6H). ${ }^{13} \mathrm{C}$ NMR (100 MHz, Acetone- $\left.d_{6}\right) \delta$ 171.3, 138.1, 138.0, 137.1, 136.2, 135.4, 130.9, 129.4, 128.9, 126.3, 124.5, 47.5, 39.6, 37.3, 37.0, 34.2, 32.9, 28.6, 21.3, 19.3, 12.1, 12.0. MALDI-TOF m/z, $\mathrm{M}^{+}$calcd. for $\mathrm{C}_{23} \mathrm{H}_{36} \mathrm{~N}_{2} \mathrm{O}$ : 356.283 ; found: 356.734 .

(S)-3-(aminomethyl)-N-((2E,4E,6E,8E)-3,7-dimethyl-9-(2,6,6-trimethylcyclohex-1-en-1-yl)nona2,4,6,8-tetraen-1-yl)-5-methylhexanamide (3k, Ret-Pregabalin), yield $25.8 \%$ (220 mg). ${ }^{1} \mathrm{H}$ NMR (400 $\left.\mathrm{MHz}, \mathrm{CDCl}_{3}\right) \delta 6.80(\mathrm{~s}, 1 \mathrm{H}), 6.58(\mathrm{dd}, J=15.1,11.3 \mathrm{~Hz}, 1 \mathrm{H}), 6.24(\mathrm{~d}, J=15.2 \mathrm{~Hz}, 1 \mathrm{H}), 6.16-6.01(\mathrm{~m}$, $3 \mathrm{H}), 5.48(\mathrm{t}, J=7.0 \mathrm{~Hz}, 1 \mathrm{H}), 4.09-3.87$ (m, 2H), 3.63 (bs, 2H), 2.70 (ddd, $J=19.2,12.7,5.4 \mathrm{~Hz}, 2 \mathrm{H})$, 2.23 (ddd, $J=19.5,14.0,6.6 \mathrm{~Hz}, 2 \mathrm{H}), 2.01(\mathrm{t}, J=6.3 \mathrm{~Hz}, 2 \mathrm{H}), 1.95$ (s, 3H), $1.85(\mathrm{~s}, 3 \mathrm{H}), 1.71(\mathrm{~s}, 3 \mathrm{H})$, $1.67-1.56(\mathrm{~m}, 3 \mathrm{H}), 1.50-1.40(\mathrm{~m}, 2 \mathrm{H}), 1.18(\mathrm{dd}, J=11.2,7.0 \mathrm{~Hz}, 2 \mathrm{H}), 1.02(\mathrm{~s}, 6 \mathrm{H}), 0.89$ (d, $J=6.5$ $\mathrm{Hz}, 6 \mathrm{H}) .{ }^{13} \mathrm{C}$ NMR $\left(101 \mathrm{MHz}, \mathrm{CDCl}_{3}\right) \delta 172.5,138.0,137.8,137.3,136.3,136.3,130.3,129.4,127.5$, 126.9, 125.0, 44.7, 41.8, 39.8, 39.6, 37.8, 35.2, 34.4, 33.2, 29.2, 25.4, 23.0, 22.9, 22.0, 19.5, 12.9, 12.8. MALDI-TOF $m / z \mathrm{M}^{+}$calcd. for $\mathrm{C}_{28} \mathrm{H}_{46} \mathrm{~N}_{2} \mathrm{O}: 426.361$; found: 426.801 .

(S)-2-(2-aminoacetamido)- $N$-((2E,4E,6E,8E)-3,7-dimethyl-9-(2,6,6-trimethylcyclohex-1-en-1-yl)nona2,4,6,8-tetraen-1-yl)-3-methylbutanamide (3l, Ret-L-Val-Gly): yield 50.2\% (443 mg); ${ }^{1} \mathrm{H} \mathrm{NMR}$ (400 MHz, Acetone- $\left.d_{6}\right) \delta 8.06(\mathrm{~d}, J=9.2 \mathrm{~Hz}, 1 \mathrm{H}), 7.73(\mathrm{t}, J=5.4 \mathrm{~Hz}, 1 \mathrm{H}), 6.64(\mathrm{dd}, J=15.1,11.2 \mathrm{~Hz}, 1 \mathrm{H}), 6.29(\mathrm{~d}$, 
$J=15.2 \mathrm{~Hz}, 1 \mathrm{H}), 6.24-6.03(\mathrm{~m}, 3 \mathrm{H}), 5.53(\mathrm{t}, J=7.0 \mathrm{~Hz}, 1 \mathrm{H}), 4.38(\mathrm{~d}, J=6.4 \mathrm{~Hz}, 1 \mathrm{H}), 3.96(\mathrm{dd}, J=$ 10.9, $5.1 \mathrm{~Hz}, 2 \mathrm{H}), 3.74(\mathrm{~d}, J=7.8 \mathrm{~Hz}, 2 \mathrm{H}), 2.00(\mathrm{t}, J=6.1 \mathrm{~Hz}, 2 \mathrm{H}), 1.94(\mathrm{~d}, J=0.9 \mathrm{~Hz}, 3 \mathrm{H}), 1.86(\mathrm{~d}, J=$ $0.9 \mathrm{~Hz}, 3 \mathrm{H}), 1.68(\mathrm{~d}, J=0.8 \mathrm{~Hz}, 3 \mathrm{H}), 1.62-1.56(\mathrm{~m}, 2 \mathrm{H}), 1.50-1.41(\mathrm{~m}, 2 \mathrm{H}), 1.01(\mathrm{~s}, 6 \mathrm{H}), 0.90(\mathrm{dd}, J$ $=10.8,6.8 \mathrm{~Hz}, 6 \mathrm{H}) .{ }^{13} \mathrm{C}$ NMR $\left(100 \mathrm{MHz}\right.$, Acetone- $\left.d_{6}\right) \delta 170.73,170.31,138.17,137.98,137.05,136.55$, $135.53,130.89,128.89,128.76,126.34,124.68,57.56,54.15,39.66,37.21,34.21,32.92,31.84,28.67$, 21.35, 19.29, 19.09, 17.82, 12.15, 12.05. MALDI-TOF $m / z[\mathrm{M}+\mathrm{Na}]^{+}$calcd. for $\mathrm{C}_{27} \mathrm{H}_{43} \mathrm{~N}_{3} \mathrm{O}_{2} \mathrm{Na}$ : 464.325; found: 463.824 .

(S)-2-amino- $N$-(2-(((2E,4E,6E,8E)-3,7-dimethyl-9-(2,6,6-trimethylcyclohex-1-en-1-yl)nona-2,4,6,8tetraen-1-yl)amino)-2-oxoethyl)-3-methylbutanamide (3m, Ret-Gly-L-Val): yield 52.9\% (467 mg); ${ }^{1} \mathrm{H}$ NMR (400 MHz, $\left.\mathrm{CDCl}_{3}\right) \delta 8.07$ (t, $\left.J=5.5 \mathrm{~Hz}, 1 \mathrm{H}\right), 7.09$ (t, $\left.J=5.3 \mathrm{~Hz}, 1 \mathrm{H}\right), 6.57$ (dd, $J=15.1,11.3 \mathrm{~Hz}$, $1 \mathrm{H}), 6.29-6.01(\mathrm{~m}, 4 \mathrm{H}), 5.46(\mathrm{t}, J=7.0 \mathrm{~Hz}, 1 \mathrm{H}), 3.99(\mathrm{dd}, J=15.2,5.8 \mathrm{~Hz}, 4 \mathrm{H}), 3.25(\mathrm{~d}, J=4.1 \mathrm{~Hz}$ $1 \mathrm{H}), 2.28-2.16(\mathrm{~m}, 1 \mathrm{H}), 2.01(\mathrm{t}, J=6.4 \mathrm{~Hz}, 3 \mathrm{H}), 1.95(\mathrm{~s}, 3 \mathrm{H}), 1.90(\mathrm{~d}, J=4.1 \mathrm{~Hz}, 1 \mathrm{H}), 1.84(\mathrm{~s}, 3 \mathrm{H}), 1.71$ $(\mathrm{d}, J=0.5 \mathrm{~Hz}, 3 \mathrm{H}), 1.65-1.56(\mathrm{~m}, 2 \mathrm{H}), 1.48-1.43(\mathrm{~m}, 2 \mathrm{H}), 1.30-1.25(\mathrm{~m}, 1 \mathrm{H}), 1.02(\mathrm{~s}, 6 \mathrm{H}), 0.97(\mathrm{~d}$, $J=6.9 \mathrm{~Hz}, 4 \mathrm{H}), 0.83(\mathrm{~d}, J=6.9 \mathrm{~Hz}, 3 \mathrm{H}) .{ }^{13} \mathrm{C} \mathrm{NMR}\left(100 \mathrm{MHz}, \mathrm{CDCl}_{3}\right) \delta 175.6,169.1,138.0,137.8,137.4$, $136.3,136.2,130.2,129.4,126.9,126.9,125.1,60.3,43.3,39.8,37.9,34.4,33.2,31.3,29.2,22.0,19.8$, 19.5, 16.5, 12.9, 12.9. MALDI-TOF $m / z$ [M+Na] $]^{+}$calcd. for $\mathrm{C}_{27} \mathrm{H}_{43} \mathrm{~N}_{3} \mathrm{O}_{2} \mathrm{Na}$ : 464.325; found: 463.810 .

2-amino- $N$-(2-(((2E,4E,6E,8E)-3,7-dimethyl-9-(2,6,6-trimethylcyclohex-1-en-1-yl)nona-2,4,6,8tetraen-1-yl)amino)-2-oxoethyl)acetamide (3n, Ret-Gly-Gly): yield 17.2\% (137 mg); ${ }^{1} \mathrm{H} \mathrm{NMR} \mathrm{(400} \mathrm{MHz,}$ Acetone- $\left.d_{6}\right) \delta 8.28-8.09(\mathrm{~m}, 1 \mathrm{H}), 7.77(\mathrm{t}, J=5.5 \mathrm{~Hz}, 1 \mathrm{H}), 6.63(\mathrm{ddd}, J=24.1,15.1,11.2 \mathrm{~Hz}, 1 \mathrm{H}), 6.26$ $(\mathrm{d}, J=15.2 \mathrm{~Hz}, 1 \mathrm{H}), 6.21-6.00(\mathrm{~m}, 3 \mathrm{H}), 5.49(\mathrm{t}, J=6.8 \mathrm{~Hz}, 1 \mathrm{H}), 3.97-3.88(\mathrm{~m}, 2 \mathrm{H}), 3.85(\mathrm{dd}, J=3.9$, $1.7 \mathrm{~Hz}, 1 \mathrm{H}), 3.37$ (s, 2H), $3.30-3.20(\mathrm{~m}, 2 \mathrm{H}), 2.70$ (d, J=5.1 Hz, 2H), $1.98(\mathrm{~d}, J=4.9 \mathrm{~Hz}, 2 \mathrm{H}), 1.93-$ $1.91(\mathrm{~m}, 3 \mathrm{H}), 1.82(\mathrm{~d}, J=0.9 \mathrm{~Hz}, 3 \mathrm{H}), 1.62-1.54(\mathrm{~m}, 3 \mathrm{H}), 1.50-1.38(\mathrm{~m}, 2 \mathrm{H}), 1.25(\mathrm{~s}, 2 \mathrm{H}), 0.98(\mathrm{~d}, J$ $=3.4 \mathrm{~Hz}, 6 \mathrm{H}) .{ }^{13} \mathrm{C}$ NMR $\left(100 \mathrm{MHz}\right.$, Acetone- $\left.d_{6}\right) \delta 171.1,168.9,138.2,138.0,137.1,136.3,135.4,130.9$, $129.0,128.9,126.3,124.6,54.2,47.0,39.7,34.2,32.9,28.7,26.8,25.1,21.4,19.3,12.2,12.1$. MALDI-

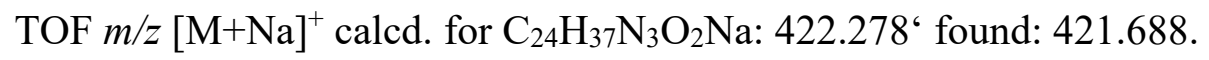

The synthetic scheme for emixustat-L-Val-Gly (EVG) is shown in Fig. 2 (above). Racemic emixustat was synthesized according to reported methods ${ }^{2}$. Emixustat-VG was synthesized as following: Racemic emixustat (526 mg, 2.0 mmol, 1.0 equiv.) Fmoc-Gly-Val (1981 mg, 5.0 mmol, 2.5 equiv.), 1Ethyl-3-(3-dimethylaminopropyl)carbodiimide $\quad($ EDC) $\quad(383 \quad \mathrm{mg}, \quad 2.0 \mathrm{mmol}, \quad 1.0 \quad$ equiv.), 
hydroxybenzotriazole (HOBt) $(270 \mathrm{mg}, 2.0 \mathrm{mmol}, 1.0$ equiv.) and N, N- diisopropylethylamine $(517 \mathrm{mg}$, $4.0 \mathrm{mmol}, 2.0$ equiv.) were dissolved in dry DMF $(20 \mathrm{~mL})$ at room temperature. Stirring was continued at room temperature for $2 \mathrm{~h}$; then the reaction was quenched with $10 \% \mathrm{~K}_{2} \mathrm{CO}_{3}$ aqueous solution. The solution was stirred for another $1 \mathrm{~h}$ to allow hydrolysis of the ester bond. The intermediate Fmoc-protected emixustat conjugate (emixustat-VG-Fmoc) was extracted by ethyl acetate and washed with brine and dried over $\mathrm{Na}_{2} \mathrm{SO}_{4}$. The crude product was purified by silica gel column chromatography, with hexane and ethyl acetate as eluant. $10 \mathrm{~mL} 20 \%$ piperidine in ethyl acetate was added to emixustat-VG-Fmoc to remove Fmoc. The crude product was purified by silica gel chromatography.

(2S)-2-(2-aminoacetamido)- $N$-(3-(3-(cyclohexylmethoxy)phenyl)-3-hydroxypropyl)-3 methylbutanamide (EVG, emixustat-L-Val-Gly) yield $27.8 \%$ (233 mg); ${ }^{1} \mathrm{H}$ NMR (400 MHz, $\left.\mathrm{CDCl}_{3}\right) \delta$. 7.23-7.18(m, 1H), $7.06(\mathrm{~s}, 1 \mathrm{H}), 6.88-6.76(\mathrm{~m}, 3 \mathrm{H}), 4.68-4.67(\mathrm{~m}, 1 \mathrm{H}), 4.18(\mathrm{~s}, 1 \mathrm{H}), 3.73(\mathrm{~d}, \mathrm{~J}=4 \mathrm{~Hz}, 2 \mathrm{H})$, $3.55-3.27(\mathrm{~m}, 3 \mathrm{H}), 2.18-2.15(\mathrm{~m}, 1 \mathrm{H}), 1.97-1.68(\mathrm{~m}, 9 \mathrm{H}), 1.33-1.17(\mathrm{~m}, 3 \mathrm{H}), 1.07-0.89(\mathrm{~m}, 8 \mathrm{H}) .{ }^{13} \mathrm{C} \mathrm{NMR}$

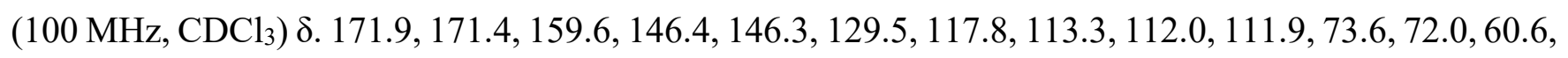
58.9, 38.7, 37.9, 37.1, 36.9, 30.9, 30.1, 26.7, 26.0, 21.2, 19.6, 18.5, 14.4. MALDI-TOF $m / z[\mathrm{M}+\mathrm{Na}]^{+}$calcd. for $\mathrm{C}_{24} \mathrm{H}_{37} \mathrm{~N}_{3} \mathrm{O}_{2} \mathrm{Na}: 442.268$; found: 441.550 .

RVG and Ret-NH2 Stability for storage. The stability of RVG prodrug was evaluated under room temperature. RVG methanol solution ( $10 \mu \mathrm{L}$ of $50 \mathrm{mg} / \mathrm{mL}$ ) was added to glass tubes. Then, methanol was removed under vacuum. Total of 9 tubes of samples were prepared and stored under vacuum in a desiccator at room temperature. For each test points (1, 4 and 8 weeks), $5 \mathrm{~mL}$ of methanol was added to the tube to dissolve RVG. The samples were then analyzed using HPLC, and each time point for each group, three samples were analyzed. $\mathrm{Ret}-\mathrm{NH}_{2}$ was used as a control, stored and tested at same condition. The degradation rate was calculated based on the HPLC results. Ion-pairing reverse-phase HPLC (RPHPLC) was performed on an Agilent 1100 series HPLC system fitted with a Beckman Coulter Ultrasphere ODS $4.6 \mathrm{~mm} \times 25 \mathrm{~cm}, 5 \mu \mathrm{m}$ pore size column. HPLC conditions: mobile phase of $\mathrm{H}_{2} \mathrm{O}$ : acetonitrile (40:60, $v: v$ ) with $0.05 \%$ trifluoroacetic acid; flow rate of $1.0 \mathrm{~mL} / \mathrm{min}$ and $\mathrm{UV}$ detector set at $325 \mathrm{~nm}$. 


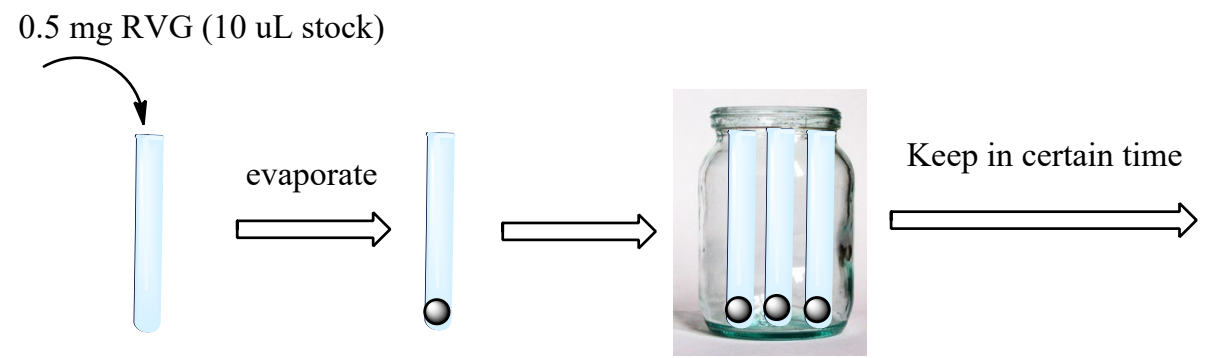

$5 \mathrm{~mL} \mathrm{MeOH}$
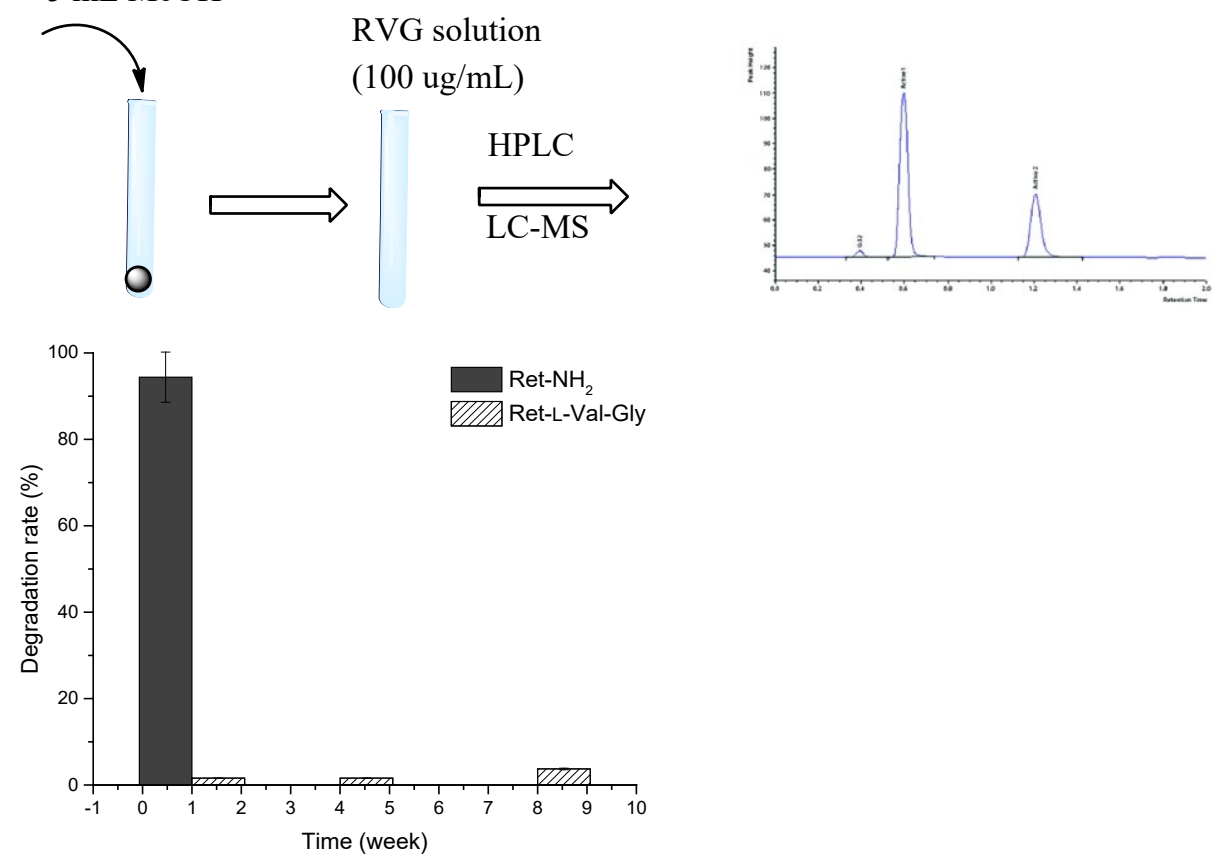

Figure S3. Procedure for assessing stability of RVG and extent of degradation of RVG during storage under vacuum at room temperature for the time periods indicated $(n=3)$. 


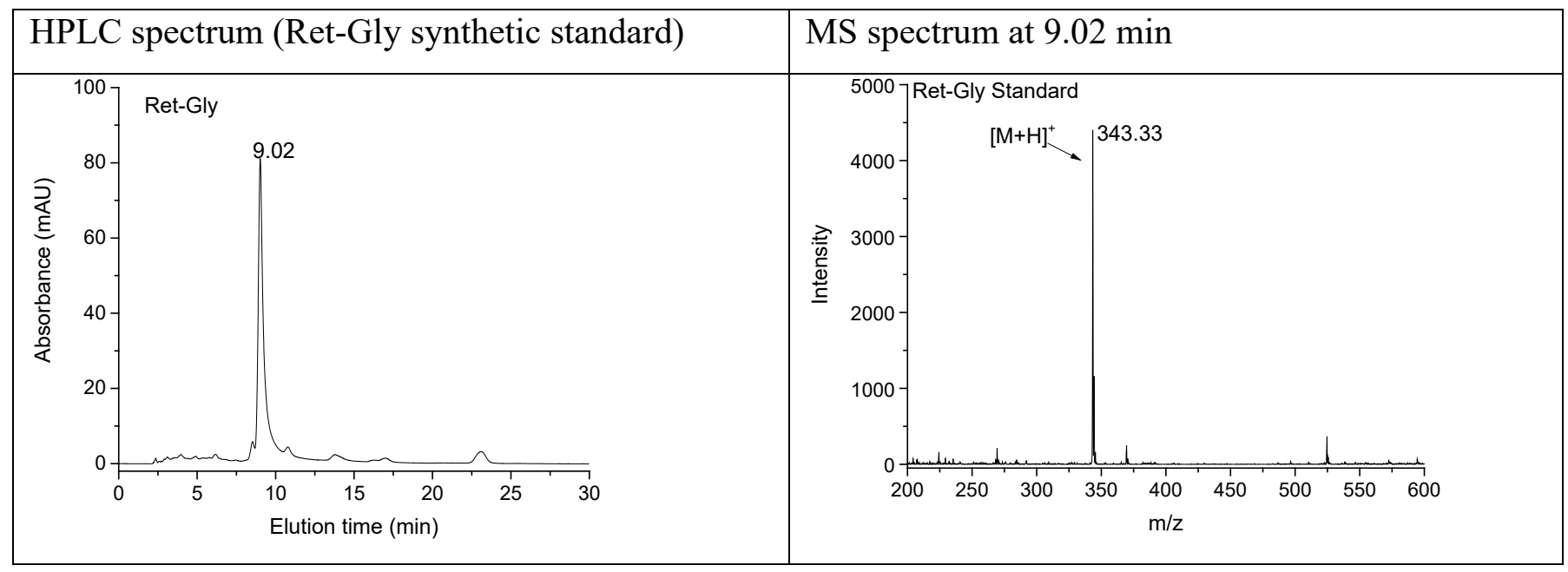

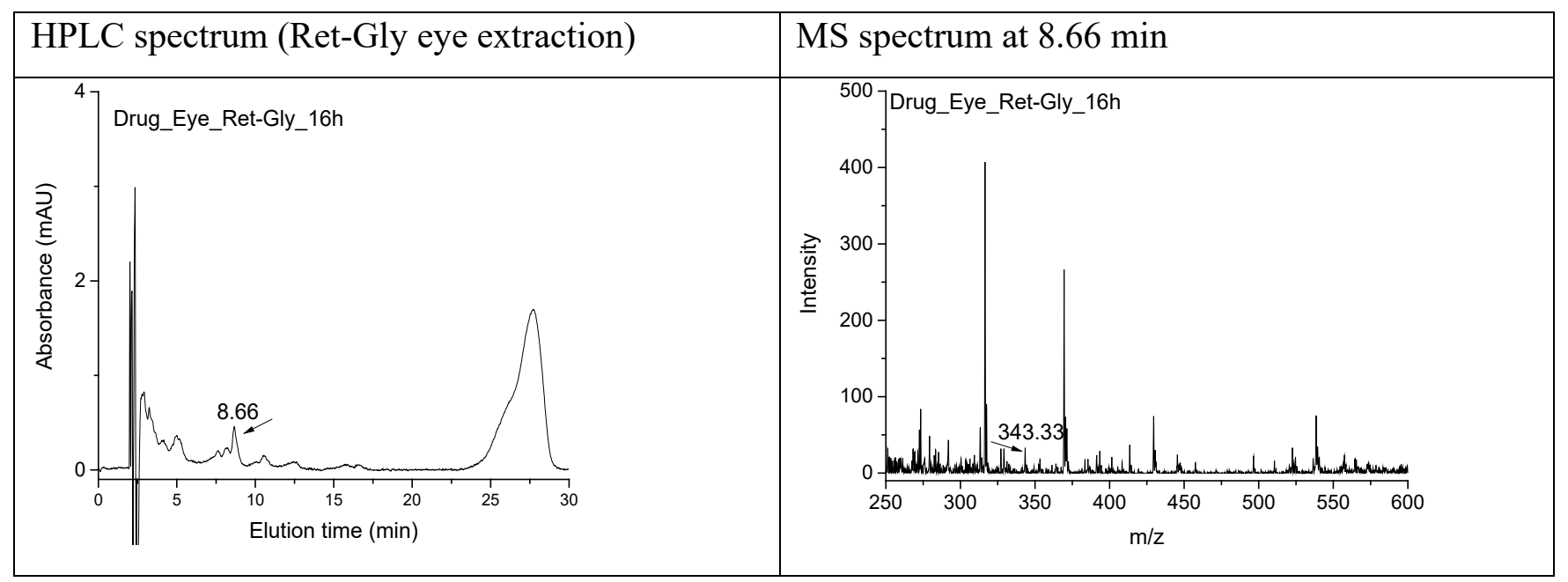

Figure S4. LC-MS analyses of the Ret- $\mathrm{NH}_{2}$ derivatives extracted from the eye, after oral administration.

\section{Reference}

(1) Golczak, M., Kuksa, V., Maeda, T., Moise, A. R., and Palczewski, K. (2005) Positively charged retinoids are potent and selective inhibitors of the trans-cis isomerization in the retinoid (visual) cycle. Proc Natl Acad Sci U S A 102, 8162-8167.

(2) Scott, I. L., Kuksa, V. A., Orme, M. W., Little, T., Gall, A., and Hong, F. (2009) pp 360pp., Acucela Inc., USA. 